\title{
Commentary: Usability in vivo
}

\section{Stuart Reeves ${ }^{1}$}

${ }^{1}$ University of Nottingham

\section{Author's Mini-bio:}

Stuart Reeves (stuart@tropic.org.uk, http://www.cs.nott.ac.uk/ str) is EPSRC Senior Research Fellow in the Mixed Reality Lab in the School of Computer Science at the University of Nottingham. He researches social and collaborative technologies, investigating how people use diverse kinds of interactive devices and systems in real world situations and places. As EPSRC Fellow (grant no. EP/K025848/1) he is exploring the connections between academic HCI research and the work of practitioners in UX and design professions. He is also author of the book Designing Interfaces in Public Settings. 
In this commentary I discuss Noam Tractinsky's article The Usability Construct: A Dead End? (HumanComputer Interaction VOL [YEAR], PAGES), which provides a thoughtful meditation on the notion of 'usability'. Usability is a troublesome matter, Tractinsky argues, resulting in over 30 years of scholarly debate in HCI that has left research communities with considerable difficulties in "reaching agreement on what this term [usability] actually means" (ibid., p. 3). Of course, this is worrying stuff for research practices that style themselves on the sciences (natural or otherwise): consolidating and reaching agreement upon things is often considered a hallmark of operating as a "normal science" (Kuhn, 1970) which, unless fixed, "limits the ability to generalize and to accumulate knowledge" (Tractinsky, p. 47). Giving evidence, Tractinsky points to the range of complex and perhaps confused ways in which usability has been discussed in HCI research. In using the explanatory mechanism of the "umbrella construct", Tractinsky dismantles and charts the different cycles or stages that such constructs like usability go through in their existence as objects of interest within the formal exchanges of academic research (i.e., the literature - a point I will return to shortly when discussing formal accounts).

Usability, as construct, is thus presented as a matter for 'scientific' ${ }^{\text {l }}$ attention. Doing this is undergirded by a desire for upholding greater theoretical standardisation across HCI communities around notions like usability; Tractinsky would not be alone in wishing for such a thing (e.g., Whittaker, Terveen, and Nardi (2000)). The problem is that "HCI is fundamentally a constructive activity and that activity in itself can presumably change the nature and meaning of the usability construct"-thus the constitution of HCI seems to wilfully work against the idea of normal science and establishing agreements around matters seemingly so critical as usability or core phenomena like interaction (e.g., see Hornbæk and Oulasvirta (2017)). In that sense the analytic solution for usability being offered in the paper is the adoption of umbrella constructs.

Tractinsky advances the idea that we need a stronger scientific HCI focussed on better understanding of constructs like usability along with its constituent components. Doing so, he argues, might also help to provide HCI practitioners ${ }^{2}$ with better, more reliable methods to move forwards and develop their own work. One option here would be in better "explicating the relations between design choices and design outcomes and getting rid of a confounding usability construct" (ibid., p. 49). Such an improvement to the theoretical landscape of HCI, he argues, is connected to and would support corresponding advances in the practical work of designing interactive systems by practitioners.

\section{Are we talking about the same 'usability'?}

I want us to be a bit more careful about what we mean when we say 'usability' here. To start, I think I can agree the following: usability certainly seems to be a problem when we speak of it in terms of a

\footnotetext{
${ }^{1}$ I use quotation marks here not in order to render the use of 'scientific' ironic but rather to indicate the lack of clarity over what kind of science we are talking about here. Tractinsky does make some initial moves: "the goal of scientific inquiry is to understand the world: this includes not just physical and natural phenomena but also human and social perceptions, behaviour and artifacts" (p. 6) - which seem to suggest a unified field of inquiry with shared methods and principles.

${ }^{2}$ By which I presume is meant something along the lines of UX, Interaction Design, etc. practitioners. Although like much talk of 'practitioners' in the HCI literature, it is often unclear and seems to gloss a diverse set of potential activities and roles.
} 
scientific version of HCI, as Tractinsky ably documents. However, this argument here relies on a very particular conception of disciplinary knowledge formation in HCI that is not necessarily uniformly agreed upon within its cross-cutting communities. Although I would personally prefer to exercise quite significant caution around the idea of 'scientising' HCI, that particular issue is not the topic of this commentary (I have addressed it elsewhere, see Reeves (2015a) and Reeves (2015b)).

Instead my interest instead is in the following. Elements of Tractinsky's argument seem to rely upon ideas of a seamlessness between the concerns of HCI research and practitioners' work. Now, it's of course true that a focus issue for practitioners is most definitely usability (perhaps ostensibly). This is testified to by the persistent popularity of perhaps the most clear manifestation, usability testing, which remains a key part of user research even as design remit for practitioners has (at least in the discourse of practice) expanded well beyond 'usability'. But having spent time observing practitioners at work performing usability testing, I am left wondering if usability-in-practice really is a 'problem'. In other words, while usability may present itself as a problematic matter on a conceptual level to Tractinsky's sense of scientific HCI, I find it hard to locate any similar order of conceptual trouble encountered by practitioners in their work practices ${ }^{3}$. To explain this another way, conceptual troubles about usability do not seem to be topicalised during testing (as far as I can tell). This is not to suggest an absence of debate about usability and usability testing practices and indeed best practices amongst practitioners of various stripes; on the contrary, there are frequent and lively debates about topics such as how many users count as 'enough' (Nielsen and Landauer, 1993), how much moderators should intervene, to what degree participants should perform think-aloud, and the differences between remote and local testing (to name a few). But these are fundamentally not conceptual debates akin to that described by Tractinsky.

To say it differently again, I'm simply suggesting the nature of problems faced by practitioners are not necessarily the same as those faced in academic excursions to addressing usability (an observation which, surely, is hardly controversial?). Case in point: usability cannot be that much of a mystery to many practitioners. As professionals during usability testing, practitioners - UXers, designers, client stakeholders, developers, product managers, etc._-routinely go about identifying 'this' aspect or 'that' aspect of (observed or inspected) use, and formulate (and agree upon) these usability problems in course of their activities. Of course none of this is to suggest that identifying usability problems is somehow uncomplicated or that perhaps the existence, priority or importance of identified problems is uncontested. Rather, my point is that while techniques like usability testing do indeed benefit from experience and manicured technical and 'soft' skills, locating usability problems in the collective courses of work of practitioners is not a vexed matter at some conceptual or fundamental level or subject to the kind of serious and quite fundamental intellectual disagreement documented in the HCI research literature and pointed to by Tractinsky. In contrast, practitioners' lab testing largely takes place in an orderly fashion, production of usability findings follow, designers, developers, managers, etc. act upon findings, and improvements to products are made (and evaluated in some way, e.g., 'Key Performance Indicators' or 'Return On Investment' models, for instance, as noted in Tractinsky's article, p. 49). In sum it's quite hard to see similar conceptual crises within the pragmatics of this kind of work to that laid out in The Usability Construct: A Dead End?.

Why might this be? On one level, it is probably the case that what is at stake differs between different uses of and senses in which we say 'usability': for practitioners, usability is lodged in the

\footnotetext{
${ }^{3}$ That said, it is indeed conceivable that such practitioners could benefit from academic researchers refining usability.
} 
context of material changes products that result in improved product outcomes (in some way), whereas for academic, HCI-scientific senses of usability, the stakes are located in pursuit of academic outcomes such as research papers, 'contributions to knowledge', continuation of research funding, and so forth. Immediately then we see that our discussion of 'usability' needs to tread carefully to avoid category mistakes; that is, confusing one sense of usability for another as described above.

\section{Formal accounts of usability and usability in vivo}

Above I made an initial foray into the idea of a dissonance in thinking about usability as a problem. Here I want to expand this to think about related aspects of this that benefit from being thrown into relief.

Specifically, I think there are differences between formal accounts of usability and the ways in which usability is lodged in practice. By formal accounts I mean definitions of usability that we find in research literature including in this paper; sometimes these might be described as 'theory'. Tractinsky's paper is ultimately a review of these formal accounts and the ways in which they fail to reach agreement on what usability is. Disagreement spans usability's constituent elements (like effectiveness, efficiency, and satisfaction as per ISO 9241-11) and causal relations between those elements (e.g., that "usability is not simply an attribute of the product, but is also a context-dependent quality" (Tractinsky, p. 21)). We also need to remind ourselves that formal accounts are of course not exclusive to academic research; for instance, there are plenty of practitioners' own articulations of usability, centred primarily around testing (e.g., see those of Krug (2010) or Kuniavsky (2003)).

The distinction here is that formal accounts (necessarily) contrast with what we might call in vivo, practical orientations to usability, like those that might be found in actual sites of work where usability becomes a foremost practical concern - usability testing as I described above being one such location. In these, usability is something to be discovered. Usability must come to be constituted as recognisable and reportable things for practitioners via "situated practices of looking-and-telling" (Garfinkel, 1967, p. 1) - as sharedly objective ${ }^{4}$ matters agreed upon between practitioners in the course of their examinations of prototypes, competitor products, or any other relevant artefact under inspection. It is not that formal accounts of usability are somehow irrelevant here, but rather their relationship is more complex, standing in as resources that orient and shape - but do not constitute-what counts as usability.

Expanding this last point, usability-in-practice cannot necessarily be readily, formally specified beforehand. This is perhaps one of the key reasons for actually even doing usability testing, where troubles-in-use unfold as identifiable socially shared objects, 'filled in' by the interactional work of moderators and test participants. While many of these troubles follow paths that are familiar to moderators and perhaps others observing (e.g., participants cannot work out what to do next), they are always contingent on local exigencies. In other words this is a sense of usability as discovered interactionally ${ }^{5}$ through the concrete, concerted, organised actions of participants, moderators, observers, stakeholders (etc.) that are done during a test, rather than as a priori, theorised sets of

\footnotetext{
4 The sense of 'objective' here is that of the achievement of seeing something together, as a social matter, rather than in reference to philosophical discussions about subjective-objective dualism.

${ }^{5}$ To say this another way, practitioners work with a typology of usability in a Schützian sense.
} 
usability criteria. As the process of testing develops from one moment to the next, moderators and observers must do interactional work to transform the stream of test 'happenings' into noticeable cases of usability issues ${ }^{6}$. This is not trivial of course: some problems visibly encountered by test participants may not be relevant for a wide range of reasons even though they seem to be possible usability troubles (in which case do they then 'count' as usability issues at all?).

Overall it's not clear if the umbrella construct takes such a distinction into account at all - rather, it seems geared to managing conceptual development entirely within academic discourse and not much of the lived work of usability. This is not a problem per se, but rather an oversight that could benefit from inspection, i.e., research on the praxeology of usability as complement to the kind of formal development of usability as umbrella construct. This means that I think there are alternate (not alternative) perspectives on usability to be found embedded in the practices of practitioners, a sense of usability-in-practice that emerges from interactional phenomena themselves. It's perhaps in this sense that we might also look to see what usability means for them, as an autochthonous artefact of the concrete circumstances of practitioners' work.

\section{References}

Garfinkel, H. (1967). Studies in Ethnomethodology. Polity Press.

Garfinkel, H., Lynch, M., \& Livingston, E. (1981). The Work of a Discovering Science Construed with Materials from the Optically Discovered Pulsar. Philosophy of the Social Sciences 11 (June 1981), Sage, 131-158. https://doi.org/10.1177/004839318101100202

Hornbæk, K., \& Oulasvirta, A. (2017). What is interaction? In Proceedings of the SIGCHI Conference on Human Factors in Computing Systems (May 2017), ACM Press.

Krug, S. (2010). Rocket Surgery Made Easy. New Riders.

Kuhn, T. S. (1970). The Structure of Scientific Revolutions (Second Edition). Chicago: University of Chicago Press.

Kuniavsky, M. (2003). Observing the user experience. Morgan Kaufman

Nielsen, J., \& Landauer, T. K. (1993). A mathematical model of the finding of usability problems. In Proceedings of the INTERACT '93 and CHI '93 Conference on Human Factors in Computing Systems (CHI '93). ACM, New York, NY, USA, 206-213.

http://dx.doi.org/10.1145/169059.169166

Reeves, S. (2015a). Locating the 'big hole' in HCI research. interactions 22, 4 (June 2015), 5356. http://dx.doi.org/10.1145/2785986

\footnotetext{
${ }^{6}$ In some ways rather like that of Garfinkel, Lynch and Livingston's astronomers (1981) discovering an optical pulsar but certainly not the same as the particulars of their astrophysical work practices.
} 
Reeves, S. (2015b). Human-computer interaction as science. In Proceedings of The Fifth Decennial Aarhus Conference on Critical Alternatives (August 2015), AA '15, Aarhus University Press, pp. 73-84. http://dx.doi.org/10.7146/aahcc.v1i1.21296

Whittaker, S., Terveen, L., \& Nardi, B. A. (2000). Let's stop pushing the envelope and start addressing it: a reference task agenda for HCI. Hum.-Comput. Interact. 15, 2 (September 2000), 75-106. http://dx.doi.org/10.1207/S15327051HCI1523 2 